\title{
Açóes de terapeutas ocupacionais na atençáo à pessoa com problemas relacionados ao uso de álcool e outras drogas ${ }^{1}$
}

\author{
Adriana Maria Nogueira ${ }^{\mathrm{a}, \mathrm{b}}$, Andrea Ruzzi Pereira ${ }^{\mathrm{c}, \mathrm{d}}$ \\ ${ }^{a}$ Centro de Atenção Psicossocial - CAPS Luis Giani, Sacramento, MG, Brasil \\ bDependência Química e Gestão da Assistência Terapêutica, Universidade Cândido Mendes - UCAM, \\ Campos dos Goytacazes, RJ, Brasil \\ 'Programa em Saúde na Comunidade, Faculdade de Medicina de Ribeirão Preto - FMRP, Universidade de São \\ Paulo - USP, Ribeirão Preto, SP, Brasil \\ ${ }^{d}$ Universidade Federal do Triângulo Mineiro - UFTM, Uberaba, MG, Brasil
}

\begin{abstract}
Resumo: Neste estudo exploratório descritivo buscou-se caracterizar as intervenções de terapeutas ocupacionais em espaços destinados ao atendimento de pessoas com problemas relacionados ao uso de álcool e outras drogas, identificando a forma e frequência dos atendimentos e mapeando as principais atividades desenvolvidas e os seus objetivos. Os sujeitos da pesquisa foram captados por meio da Técnica Bola de Neve, participando 11 terapeutas ocupacionais atuantes em sete estados da federação. A coleta de dados ocorreu de outubro a dezembro de 2011 por meio de questionário estruturado autoexplicativo e autoaplicável, elaborado pelas autoras, o qual indagou ao profissional entrevistado sobre sua formação (graduação, especializações/títulos, tempo de atuação na área da dependência química) e sobre sua atuação profissional (forma e frequência dos atendimentos, critérios que norteiam a escolha da forma e frequência dos atendimentos, atividades mais utilizadas nos atendimentos e principais objetivos a serem alcançados com a realização de tais atividades). Verificou-se que são realizados atendimentos individuais e grupais, com frequência de duas vezes ou mais por semana. As atividades mais desenvolvidas são as autoexpressivas; as atividades manuais; e aquelas relacionadas à educação em saúde, autocuidado e reorganização do cotidiano. Os objetivos mais relatados foram: resgatar valores pessoais e sociais; ressignificar as atividades cotidianas; ampliar a corresponsabilização da família no tratamento; expressar sentimentos e conflitos internos; e usufruir de momentos de lazer e relaxamento. Concluiu-se que existe um comportamento variado entre os profissionais com relação à sua prática, apontando-se as diferentes concepções filosóficas e metodológicas envolvidas nesse processo.
\end{abstract}

Palavras-chave: Terapia Ocupacional, Centros de Tratamento de Abuso de Substâncias, Tratamento.

\section{Actions of occupational therapists in the care of people with problems related to use of alcohol and other drugs}

\begin{abstract}
This exploratory descriptive study aimed to characterize the interventions of occupational therapists in substance abuse treatment centers, identifying the form and frequency of sessions and mapping the major activities and their objectives. The study subjects were obtained through the Snowball Sampling technique, eleven participating occupational therapists working in seven states. Data collection occurred from October to December 2011 through self-explanatory and self-administered structured questionnaires developed by the authors, which inquired the professionals interviewed about their training (undergraduate and graduate courses; time working in the field of chemical dependency) and professional performance (form and frequency of sessions, criteria governing the choice of form and frequency of sessions, activities most frequently used in the sessions, and key objectives to be achieved
\end{abstract}

Autor para correspondência: Andrea Ruzzi Pereira, Núcleo de Estudo e Pesquisa em Saúde Mental, Álcool e outras Drogas - NEPSMAD, Departamento de Terapia Ocupacional, Instituto de Ciências da Saúde, Universidade Federal do Triângulo Mineiro - UFTM, Rua Getúlio Guaritá, 159, $3^{\circ}$ Piso, CEP 38025-440, Uberaba, MG, Brasil, e-mail: nepsmad@gmail.com

Recebido em 25/9/2012; $1^{\text {a }}$ Revisão em 8/4/2013; $2^{\text {a }}$ Revisão em 4/5/2013; Aceito em 14/5/2013. 
with the development of such activities). It was possible to verify that individual and group sessions are performed of twice a week or more often. The most developed activities are self-expressive and manual activities and those related to health education, self-care, and reorganization of everyday life. The goals most frequently reported were recovery of personal and social values; reframing the activities of everyday life; expansion of the co-responsibility of families in the treatments; expression of feelings and internal conflicts; and enjoyment of relaxation and leisure moments. We conclude that varied behavior occurs among the professionals with respect to their practice, indicating the different philosophical and methodological conceptions involved in this process.

Keywords: Occupational Therapy, Substance Abuse Treatment Centers, Therapeutics.

\section{Introdução}

As pessoas que fazem uso de álcool e/ou outras drogas podem vir a apresentar diversos problemas, dentre eles a dependência química. A Organização Mundial da Saúde (2004, p. 13-14) define a dependência química como “[...] um transtorno da função cerebral ocasionado pelo consumo de substâncias psicoativas [...]", as quais afetam os padróes normais sensório-perceptivos, emocionais e motivacionais. Assim como as doenças neurológicas e psiquiátricas, inicialmente a dependência química não foi reconhecida como um transtorno das funçóes cerebrais. Porém, com o avanço tecnológico da Neurociência é possível

[...] visualizar e medir alterações na função cerebral desde o nível molecular e celular a alteraçóes em processos cognitivos complexos que ocorrem com o consumo de substâncias a curto e a longo prazo [...]. (ORGANIZAÇÃO..., 2004, p. 14)

Entretanto, essa base neurobiológica e biocomportamental não é capaz de, sozinha, justificar o intrincado processo no qual o comportamento de uso de uma determinada substância evolui para a compulsividade pelo consumo e para a dependência. Em todo esse processo fica evidenciada a presença de uma ação complexa de múltiplos fatores neurobiológicos, psicológicos e sociais (ORGANIZAÇÃO..., 2004).

Em consonância com tal princípio, a Política do Ministério da Saúde para Atenção Integral a Usuários de Álcool e Outras Drogas considera, em suas diretrizes, que

[...] a atenção psicossocial a pacientes com dependência e/ou uso prejudicial de álcool e outras drogas deve se basear em uma rede de dispositivos comunitários, integrados ao meio cultural e articulados à rede assistencial em saúde mental (BRASIL, 2003, p. 26).

Refletindo sobre a atenção integral à saúde dessas pessoas, Carvalho et al. (2011) consideram ainda que, diante dessa característica multicausal da dependência química, as intervençóes, além de utilizarem essa abordagem psicossocial, devem procurar atender às necessidades tanto do usuário quanto dos familiares.

Dessa forma, a recuperação e a reabilitação da pessoa com problemas relacionados ao uso de álcool e outras drogas envolvem um trabalho intersetorial e multiprofissional, no qual os terapeutas ocupacionais vêm ocupando um importante espaço.

Conforme nos apresentam Barros e Rodrigues (2011), esses profissionais possuem o significativo papel de auxiliar essas pessoas em seu reaprendizado ou aquisição de hábitos a serem desempenhados em sua vida cotidiana, promovendo a ampliação do seu repertório de habilidades sociais.

A Terapia Ocupacional, segundo apontam Barros, Lopes e Galheigo (2002, p. 366), é

[...] um campo de conhecimento e intervenção em saúde, em educação e na ação social, que reúne tecnologias orientadas para a emancipação e a autonomia de pessoas que, por razóes ligadas a problemáticas específicas (físicas, sensoriais, psicológicas, mentais e/ou sociais), apresentam, temporária ou definitivamente, dificuldades de inserção e participação na vida social.

As intervençóes terapêuticas ocupacionais podem ocorrer tanto de modo individual como grupal. Ballarin (2007) nos apresenta uma reflexão sobre as intervençóes grupais, destacando que a compreensão do funcionamento dos grupos é resultado da integração de diversos saberes como, por exemplo, Sociologia, Filosofia, Psicologia e Economia, caracterizando-se por variados fenômenos psíquicos que se influenciam mutuamente. Essa autora reflete ainda que, distinto dos grupos coordenados por profissionais dessas outras áreas, o terapeuta ocupacional privilegia o "fazer humano" e por meio desse estabelece o setting terapêutico.

A Terapia Ocupacional concebe as atividades humanas como

[...] constituídas por um conjunto de açôes que apresentam qualidades, demandam capacidades, materialidades e estabelecem mecanismos 
internos para sua realização. (CASTRO; LIMA; BRUNELLO, 2001, p. 47)

Essas atividades podem se desdobrar em várias fases, caracterizando-se como um processo experimental de vida para o indivíduo. Essa linguagem ativa se configura em um caminho para o autoconhecimento, para o conhecimento do outro, do mundo que nos rodeia, enfim, da nossa cultura (CASTRO; LIMA; BRUNELLO, 2001).

Essas autoras analisam que as atividades dão forma e estrutura ao fazer das pessoas, estabelecendo um sistema de relaçóes que envolvem a construção da qualidade da vida cotidiana. E a qualidade da vida cotidiana nada mais é que a percepçâo subjetiva do sujeito sobre seu bem-estar e suas condiçôes de vida.

Por tudo isso, observa-se que as açôes da Terapia Ocupacional na recuperação e reabilitação da pessoa com problemas relacionados ao uso de álcool e outras drogas estão em consonância com os princípios da reabilitaçáo psicossocial.

A Organização Mundial da Saúde e a Organização Pan-Americana de Saúde (2001, p. 62) apresentam a reabilitação psicossocial como

[...] um processo que oferece aos indivíduos que estâo debilitados, incapacitados ou deficientes em virtude de transtorno mental a oportunidade de atingir o seu nível potencial de funcionamento independente na comunidade [...]

Dessa forma, para que esse processo possa acontecer devem ser proporcionadas a essas pessoas as condiçóes necessárias para o desenvolvimento desse potencial. Nesse sentido, Saraceno (2001, p. 16) apresenta que a reabilitação psicossocial é um

[...] processo de reconstrução, um exercício pleno da cidadania, e, também, de plena contratualidade nos três grandes cenários: habitat, rede social, e trabalho com valor social,

configurando-se um modelo no qual se considera a complexidade humana sob a óptica desses diferentes fatores mutuamente influenciados.

Considerando-se que a intervenção terapêutica ocupacional pode configurar-se como uma ação possibilitadora desse processo, propóe-se neste trabalho descrever as ações realizadas por terapeutas ocupacionais na atenção à pessoa com problemas relacionados ao uso de álcool e outras drogas.

Buscou-se identificar a frequência do atendimento terapêutico ocupacional de forma individual e/ou grupal, em espaços destinados à recuperaçáo e reabilitação dessas pessoas, mapear as principais atividades utilizadas pelos terapeutas ocupacionais nesses espaços e os principais objetivos dessas atividades.

\section{Materiais e métodos}

O presente trabalho caracteriza-se como um estudo exploratório descritivo. A coleta dos dados ocorreu nos meses de outubro a dezembro de 2011, por meio de um instrumento estruturado autoexplicativo e autoaplicável, elaborado pelas autoras, o qual indagou sobre: (a) o perfil do profissional: ano e instituição da graduação, se possui especializaçóes/títulos, e há quanto tempo atua na atenção à pessoa com problemas relacionados ao uso de álcool e outras drogas; (b) a forma e frequência dos atendimentos: foram apresentadas as modalidades de atendimento individual e grupal, cada uma com quatro opçóes para serem assinaladas: "diariamente", "uma vez por semana", "duas ou mais vezes por semana" e "esporadicamente/conforme demanda"; (c) qual(is) critério(s) são utilizados para nortear a escolha da forma e frequência dos atendimentos; (d) quais as atividades mais utilizadas nos atendimentos; e (e) quais os principais objetivos de tais atividades. Esses três últimos tópicos foram apresentados em forma de perguntas abertas.

Foi realizado um pré-teste, no qual as questóes foram calibradas para atender aos objetivos do trabalho. Os dados coletados foram analisados conforme a técnica de análise de conteúdo de Bardin (2010), sendo agrupados por similaridades e encontrando-se o que os fazia divergentes e comuns, sendo então classificados e apresentados em forma de frequência absoluta e percentual.

A população participante deste trabalho foram terapeutas ocupacionais, definidos conforme os seguintes critérios de inclusão: (a) ser terapeuta ocupacional devidamente registrado no conselho de classe; (b) atuar na atenção à pessoa com problemas relacionados ao uso de álcool e outras drogas, em espaços destinados à recuperaçáo e reabilitação dessa população; (c) compreender e aceitar participar do estudo em questáo; e (d) assinar e enviar aos pesquisadores o Termo de Consentimento Livre e Esclarecido - TCLE. Os critérios deliberados para exclusão do participante foram: (a) não devolver aos pesquisadores o TCLE assinado; (b) solicitar a sua retirada da pesquisa; e (c) atuar em outro país.

A amostra dos sujeitos de pesquisa foi composta pelo método de amostragem não probabilística intencional, submetida à disposição de participação por parte dos profissionais que tiveram conhecimento 
do propósito do trabalho e entraram em contato com as pesquisadoras. A captação desses sujeitos ocorreu por meio de divulgação em comunidades virtuais de terapeutas ocupacionais do Yahoo! Grupos, que é um gerenciador de lista de discussão, acessível a partir de uma página na rede mundial de computadores, que possibilita a seus usuários criar seus próprios grupos e permitir a terceiros tornarem-se membros dele, passando os usuários, então, a trocar mensagens eletrônicas; grupos de terapeutas ocupacionais no Facebook, que também é uma página eletrônica nos moldes de uma rede social; e conselhos representativos da classe, tais como os Conselhos Regionais de Terapia Ocupacional (Crefito) e a Associação Brasileira de Terapeutas Ocupacionais.

Atualmente, de acordo o Conselho Federal de Fisioterapia e Terapia Ocupacional, encontram-se registrados em todos os Conselhos Regionais de Fisioterapia e Terapia Ocupacional 14.722 terapeutas ocupacionais em toda a federação. Porém, não se tem o registro de quantos desses respondiam a todos os critérios de inclusão desta pesquisa, nem de quantos dos que respondiam aos critérios tinham acesso aos meios nos quais ela foi divulgada ou acessavam com frequência o sítio dos respectivos Crefitos. Os profissionais que responderam ao convite eletrônico foram estimulados a divulgar a realização da pesquisa para outros profissionais atuantes na área, conforme a Técnica da Bola de Neve proposta por Flick (2004).

O desenvolvimento deste estudo seguiu todas as normas éticas norteadoras dos trabalhos que envolvem seres humanos, sendo aprovado pelo Comitê de Ética em Pesquisa da Universidade Federal do Triângulo Mineiro - UFTM, sob número 1920/2011. Os termos de esclarecimento e os instrumentos de coleta de dados foram enviados aos participantes via correio eletrônico. Esse último, após ser preenchido, foi reenviado às pesquisadoras pelo mesmo processo. O Termo de Consentimento Livre e Esclarecido foi enviado a cada participante via correio comum para ser assinado, juntamente com um envelope já selado para a sua devolução.

\section{Resultados e discussão}

\subsection{Perfil dos profissionais}

Participaram deste trabalho 11 terapeutas ocupacionais, dez do sexo feminino e um do sexo masculino, denominados P1 a P11. Tais profissionais atuam em diversos estados do Brasil, conforme discriminado: um na Bahia (9,1\%); dois no Ceará
(18,2\%); dois em Mato Grosso (18,2\%); um no Piauí (9,1\%); um no Rio de Janeiro (9,1\%); um no Rio Grande do Sul (9,1\%) e três em São Paulo (27,3\%).

De acordo com o período de tempo desde a graduação em Terapia Ocupacional, os profissionais foram assim classificados: até 2 anos: $18,2 \%-\mathrm{n}=2$; entre 4 e 6 anos: $45,4 \%-n=5$; entre 8 e 10 anos: $18,2 \%-\mathrm{n}=2$; há mais de 20 anos: $9,1 \%-\mathrm{n}=1$; há mais de 30 anos: $9,1 \%-\mathrm{n}=1$. As instituiçóes pelas quais se graduaram foram: Escola Bahiana de Medicina e Saúde Pública $(9,1 \%$ - $n=1)$; Universidade do Vale do Paraíba - Univap $(9,1 \%$ - n = 1); Rede Metodista de Educação do Sul - Instituto Porto Alegre $(9,1 \%$ - n = 1); Frasce - Faculdade de Reabilitação da Asce (Associação de Solidariedade à Criança Excepcional - Rio de Janeiro/RJ) $(9,1 \%-\mathrm{n}=1)$; Universidade de Fortaleza - Unifor $(18,2 \%$ - n $=2)$; Faculdade Santa Terezinha - Cest (Centro Educacional Santa Terezinha/Faculdade Santa Terezinha -São Luís/MA) $(9,1 \%-\mathrm{n}=1)$; Universidade do Estado do Pará $(18,2 \%-\mathrm{n}=2)$; Pontifícia Universidade Católica de Campinas $(9,1 \%-\mathrm{n}=1)$; Escola Superior de Ensino Helena Antipoff - Niterói $(9,1 \%-\mathrm{n}=1)$.

Dentre os profissionais, $81,8 \%(\mathrm{n}=9)$ possuíam título de especialistas. As áreas de especialização são: Saúde Mental (45,4\% - n = 5); Controle da Dependência Química $(9,1 \%$ - $\mathrm{n}=1)$; Coordenação de Grupos (9,1\% - n = 1); Saúde Ocupacional e Segurança do Trabalho $(9,1 \%-\mathrm{n}=1)$; e Desenvolvimento Infantil $(9,1 \%-\mathrm{n}=1)$. Um dos profissionais especialistas em Saúde Mental também possuía o título de mestre em Psicologia; o profissional especialista em Saúde Ocupacional estava se especializando em Saúde Mental; e o profissional especialista em Desenvolvimento Infantil estava cursando mestrado em Psicanálise, Saúde e Sociedade.

Quanto ao período de tempo que atua na atenção à pessoa com problemas relacionados ao uso de álcool e outras drogas, $45,4 \%$ dos profissionais $(n=5)$ tinham até 2 anos de atuaçáo; 36,4\% (n = 4), de 2 a 4 anos; 9,1\% (n = 1), 7 anos; e 9,1\% (n=1), 24 anos de atuação.

\subsection{Forma e frequência do atendimento terapêutico ocupacional}

Todos os profissionais relataram realizar atendimentos tanto individuais como grupais. A frequência dos atendimentos individuais ocorre da seguinte forma: uma vez por semana: $9,1 \%-\mathrm{n}=1$; 
duas ou mais vezes por semana: $45,4 \%-\mathrm{n}=5$; e esporadicamente, conforme a demanda apresentada pelo usuário, $45,4 \%-\mathrm{n}=5$. A frequência dos atendimentos grupais foi assim relatada pelos profissionais: ocorre diariamente: $18,2 \%-\mathrm{n}=2$; ocorre uma vez por semana: $18,2 \%-\mathrm{n}=2$; e ocorre duas ou mais vezes por semana: $63,6 \%-n=7$.

O critério utilizado para a forma de realização dos atendimentos (individual ou grupal) foi assim caracterizado: demanda expressa pelo usuário, após ser realizada avaliaçáo, foi mencionado por $81,8 \%$ dos profissionais $(\mathrm{n}=9)$; e capacidade para participação nos grupos, relacionado à medicaçáo e/ou voliçấo do usuário, foi apresentado por $27,3 \%$ $(\mathrm{n}=3)$. Um dos profissionais mencionou ainda o critério de atendimento conforme a demanda expressa pelo serviço de Terapia Ocupacional (alto número de pessoas para serem atendidas). Outro profissional relatou que o usuário é encaminhado para o atendimento individual ou grupal pelo psiquiatra, porém pode ocorrer o remanejamento quando necessário, confirmando que a demanda expressa pelo usuário é o critério predominante.

Nesse sentido, discorrendo sobre as abordagens terapêuticas ocupacionais praticadas com essa população, Barros e Rodrigues (2011, p. 188-189) nos apresentam que o atendimento individual é uma abordagem a ser utilizada "[...] para indivíduos que apresentem demanda específica, sendo impossibilitada a intervenção grupal”. O atendimento em grupos é a abordagem mais frequente, pois o grupo terapêutico ocupacional possibilita "[...] a promoção e o desenvolvimento da capacidade de resolver de forma dialética situações conflitivas", favorecendo que seus integrantes transponham as experiências vivenciadas nesse espaço para a sua prática cotidiana.

\subsection{Atividades desenvolvidas e seus principais objetivos}

Para este estudo, os terapeutas ocupacionais foram convidados a enumerar as principais atividades que desenvolviam em sua prática profissional, nos espaços destinados à recuperação e reabilitação da pessoa com problemas relacionados ao uso de álcool e outras drogas. As atividades listadas foram categorizadas, conforme sua apresentação pelos profissionais, da seguinte forma (Tabela 1): atividades caracterizadas como autoexpressivas são desenvolvidas por $72,7 \%$ dos profissionais $(\mathrm{n}=8)$; atividades manuais/artesanato/reciclagem são desenvolvidas por $63,6 \%$ dos profissionais $(\mathrm{n}=7)$; educação em saúde/auto cuidado/reorganização do cotidiano: $54,6 \%$ dos profissionais $-\mathrm{n}=6$ ); orientação às famílias: $36,4 \%$ - $\mathrm{n}=4$ ); acolhimento/ escuta terapêutica: $27,3 \%-\mathrm{n}=3$; lazer-recreação/ passeios/caminhadas: $27,3 \%-\mathrm{n}=3$ ); atividades corporais: $18,2 \%-\mathrm{n}=2$; estimulação cognitiva: $18,2 \%-\mathrm{n}=2$; passeios culturais: $18,2 \%-\mathrm{n}=2$; atividades literárias e musicais: $18,2 \%-\mathrm{n}=2$; sessão de vídeos/filmes: 9,1\% - $\mathrm{n}=1$; laborterapia:

Tabela 1. Principais atividades desenvolvidas pelos profissionais terapeutas ocupacionais nos espaços destinados ao atendimento da pessoa com problemas relacionados ao uso de álcool e outras drogas.

\begin{tabular}{|c|c|c|c|c|c|c|c|c|c|c|c|}
\hline ATIVIDADE & $P 1$ & $P 2$ & $P 3$ & $P 4$ & P5 & $P 6$ & $P 7$ & P8 & $P 9$ & $P 10$ & $P 11$ \\
\hline Acolhimento/escuta terapêutica & $\mathbf{X}$ & & & & & $\mathbf{X}$ & $\mathbf{x}$ & & & & \\
\hline Assembleia institucional & $\mathbf{X}$ & & & & & & & & & & \\
\hline Atividades autoexpressivas & & $\mathbf{X}$ & & $\mathbf{x}$ & $\mathbf{X}$ & $\mathbf{X}$ & & $\mathbf{X}$ & $\mathbf{X}$ & $\mathbf{X}$ & $\mathbf{X}$ \\
\hline Atividades corporais & & & $\mathbf{X}$ & & & & & & & & $\mathbf{X}$ \\
\hline Atividades literárias e musicais & & & & & & $\mathbf{x}$ & & & & & $\mathbf{X}$ \\
\hline Atividades manuais/artesanato/reciclagem & & $\mathbf{X}$ & $\mathbf{x}$ & & $\mathbf{X}$ & $\mathbf{X}$ & $\mathbf{X}$ & & $\mathbf{X}$ & & $\mathbf{X}$ \\
\hline Comemoração de datas especiais & & & & & & & & & $\mathbf{X}$ & & \\
\hline $\begin{array}{l}\text { Educação em saúde/autocuidado /reorganização } \\
\text { do cotidiano }\end{array}$ & $\mathbf{X}$ & & $\mathbf{x}$ & $\mathbf{x}$ & & & $\mathbf{X}$ & & $\mathbf{X}$ & $\mathbf{X}$ & \\
\hline Estimulação cognitiva & & & $\mathbf{x}$ & & & & & & $\mathbf{X}$ & & \\
\hline $\begin{array}{l}\text { Grupo de autoajuda (atendimento conjunto com } \\
\text { aa e na) }\end{array}$ & $\mathbf{X}$ & & & & & & & & & & \\
\hline Laborterapia & & & & & & & $\mathbf{X}$ & & & & \\
\hline Lazer-recreação/passeios/caminhadas & $\mathbf{X}$ & & & & & & & & $\mathbf{X}$ & $\mathbf{X}$ & \\
\hline Orientação às famiílias & $\mathbf{X}$ & & $\mathbf{x}$ & & $\mathbf{X}$ & & $\mathbf{X}$ & & & & \\
\hline Passeio cultural & & & $\mathbf{x}$ & & & & & & & $\mathbf{X}$ & \\
\hline Sessão de vídeos/filmes & & & & & & $\mathbf{x}$ & & & & & \\
\hline
\end{tabular}


$9,1 \%-\mathrm{n}=1$; comemoração de datas especiais: $9,1 \%-\mathrm{n}=1$; assembleia institucional: $9,1 \%-\mathrm{n}=1$; grupo de autoajuda, desenvolvido conjuntamente com representantes das entidades Alcoólicos Anônimos - AA e Narcóticos Anônimos - NA: $9,1 \%-\mathrm{n}=1$.

Observa-se que, considerando as principais atividades desenvolvidas pelos profissionais, eles apresentam comportamentos diferenciados em relação à diversidade de categorias de atividades oferecidas ao usuário do serviço. Enquanto alguns profissionais relatam desenvolver até seis categorias, outro menciona uma categoria, a de "atividades autoexpressivas".

Essas distintas atividades desenvolvidas no espaço terapêutico ocupacional devem proporcionar às pessoas atendidas a satisfação de suas necessidades. Nesse espaço, procura-se compor um cenário onde as pessoas possam "[...] trocar experiências, mexer com materiais, transformar e criar, compartilhar sentimentos e emoçôes [...]" (OLIVEIRA, 2006, p. 232).

Essa autora mostra ainda que tais atividades oferecem ao indivíduo a oportunidade de expressar conflitos e desejos configurando-se, portanto, uma importante forma de comunicaçáo de sua realidade interna.

A proposta da realizaçáo de atividades na clínica terapêutica ocupacional busca o estabelecimento de "[...] uma postura ativa do paciente diante de sua saúde e doença, estimulando em cada ser o desejo de lutar em prol de si mesmo". Ao estimular o "fazer humano", possibilita a construção de novos significados e sentidos, novos caminhos e novas formas de se relacionar com o mundo. Por meio desse "fazer" surge a possibilidade de

[...] vivenciar um processo criativo (com começo, meio e fim), fazer escolhas com mais sentido, expressar sentimentos, relacionar-se com a própria atividade e com as pessoas, resgatar sua identidade e sua história [...] (BARROS; RODRIGUES, 2011, p. 188).

Os profissionais terapeutas ocupacionais participantes deste trabalho foram também solicitados a especificar os principais objetivos das atividades que realizavam. Os objetivos listados foram assim caracterizados: $90,9 \%$ dos profissionais $(\mathrm{n}=10)$ relataram que resgatar valores pessoais e sociais é um dos objetivos a serem alcançados pelos usuários; e 72,7\% $(\mathrm{n}=8)$ relataram o objetivo de ressignificar as atividades cotidianas (AVDs e AIVDs). Outros objetivos relatados foram: ampliar a corresponsabilização da família no tratamento
(45,4\% dos profissionais $-\mathrm{n}=5$ ); expressar sentimentos e conflitos internos $(45,4 \%-\mathrm{n}=5)$; lazer, relaxamento, diminuição de ansiedade e da irritabilidade $(45,4 \%-\mathrm{n}=5)$; exercitar novas práticas de convivência social $(36,4 \%-\mathrm{n}=4)$; aumentar autoestima e a autoeficácia $(36,4 \%-\mathrm{n}=4)$; refletir sobre a dependência química $(27,3 \%-\mathrm{n}=3)$; refletir sobre cuidados com a saúde $(27,3 \%-\mathrm{n}=3)$; inserção social por meio de atividades geradoras de renda $(18,2 \%-\mathrm{n}=2)$; exercitar funções cognitivas como atençáo, concentração e memória $(18,2 \%-\mathrm{n}=2)$; refletir sobre a política de redução de danos $(18,2 \%$ - $\mathrm{n}=2)$; preparar para o pós-alta, esclarecendo as possibilidades e limites da internação $(9,1 \%-\mathrm{n}=1)$; participar da construção democrática do funcionamento do serviço $(9,1 \%$ - $\mathrm{n}=1)$; e ampliar a rede social a partir de modelos identificatórios positivos dos grupos AA - Alcoólicos Anônimos e NA - Narcóticos Anônimos (9,1\% - $\mathrm{n}=1)$, conforme apresenta a Tabela 2.

Pode ser observado que, assim como em relação à diversidade de categorias de atividades oferecidas ao usuário do serviço, também quanto aos objetivos os profissionais relatam esperar que os usuários alcancem, existe esse comportamento diferenciado, ou seja, enquanto determinado objetivo é listado por $90,9 \%(\mathrm{n}=10)$ dos profissionais, outro é listado por $9,1 \%(\mathrm{n}=1)$.

Destaca-se ainda quanto a esse aspecto que somente os profissionais $\mathrm{P} 7$ e $\mathrm{P} 9$ citaram a política de redução de danos como uma alternativa a ser incorporada ao processo de intervenção, a qual pode se tornar necessária quando a abstinência total não é desejada e/ou conseguida pela pessoa com problemas relacionados ao uso de álcool e outras drogas.

$\mathrm{Na}$ Política do Ministério da Saúde para Atenção Integral a Usuários de Álcool e Outras Drogas encontramos que a abstinência não pode ser a única meta estabelecida para o tratamento dessas pessoas, visto que

[...] quando se trata de cuidar de vidas humanas, temos que, necessariamente, lidar com as singularidades, com as diferentes possibilidades e escolhas que são feitas [...] (BRASIL, 2003, p. 10).

Esse documento reflete ainda que o acolhimento sem julgamento proporciona a identificação das açóes que são possíveis com cada pessoa e da demanda que ela apresenta, tornando a relação entre profissional e usuário mais efetiva. Dessa forma, estimula-se a participação e o engajamento dele nas práticas de saúde propostas pelo profissional, sem o foco na abstinência como elemento primordial para 
Tabela 2. Principais objetivos a serem alcançados pelos usuários através das atividades desenvolvidas pelos profissionais terapeutas ocupacionais.

\begin{tabular}{|c|c|c|c|c|c|c|c|c|c|c|c|}
\hline OBJETIVO & $P 1$ & $P 2$ & P3 & $P 4$ & $P 5$ & P6 & P7 & P8 & $P 9$ & $P 10$ & $P 11$ \\
\hline $\begin{array}{l}\text { Ampliar a corresponsabilizaçao da família no } \\
\text { tratamento }\end{array}$ & $\mathbf{X}$ & & $\mathbf{X}$ & & $\mathbf{X}$ & $\mathbf{X}$ & $\mathbf{X}$ & & & & \\
\hline $\begin{array}{l}\text { Ampliar a rede social com modelos identificatórios } \\
\text { positivos (grupos aa e na) }\end{array}$ & $\mathbf{X}$ & & & & & & & & & & \\
\hline Aumentar autoestima e autoeficácia & & & $\mathbf{X}$ & & & & $\mathbf{X}$ & & $\mathbf{X}$ & $\mathbf{X}$ & \\
\hline $\begin{array}{l}\text { Exercitar funções cognitivas como atenção, } \\
\text { concentração e memória }\end{array}$ & & & $\mathbf{X}$ & & & & & & $\mathbf{X}$ & & \\
\hline Exercitar novas práticas de convivência social & $\mathbf{X}$ & & & & & $\mathbf{X}$ & $\mathbf{X}$ & & $\mathbf{X}$ & & \\
\hline Expressar sentimentos e conflitos internos & & $\mathbf{X}$ & & & & $\mathbf{X}$ & & $\mathbf{X}$ & $\mathbf{X}$ & & $\mathbf{X}$ \\
\hline $\begin{array}{l}\text { Inserção social através de atividades geradoras de } \\
\text { renda }\end{array}$ & & & & & & & $\mathbf{X}$ & & $\mathbf{X}$ & & \\
\hline $\begin{array}{l}\text { Lazer, relaxamento, diminuição de ansiedade e } \\
\text { irritabilidade }\end{array}$ & $\mathbf{X}$ & & $\mathbf{X}$ & & & & $\mathbf{X}$ & & $\mathbf{X}$ & $\mathbf{X}$ & \\
\hline $\begin{array}{l}\text { Participar da construção democrática do } \\
\text { funcionamento do serviço (assembleia de usuários) }\end{array}$ & $\mathbf{X}$ & & & & & & & & & & \\
\hline $\begin{array}{l}\text { Preparar para o pós-alta (possibilidades e limites da } \\
\text { internação) }\end{array}$ & $\mathbf{X}$ & & & & & & & & & & \\
\hline Refletir sobre a dependência química & $\mathbf{X}$ & & & $\mathbf{X}$ & & $\mathbf{X}$ & & & & & \\
\hline Refletir sobre a política de redução de danos & & & & & & & $\mathbf{X}$ & & $\mathbf{X}$ & & \\
\hline Refletir sobre cuidados com a saúde & $\mathbf{X}$ & & & $\mathbf{X}$ & & $\mathbf{X}$ & & & & & \\
\hline Resgatar valores pessoais e sociais & & $\mathbf{X}$ & $\mathbf{X}$ & $\mathbf{X}$ & $\mathbf{X}$ & $\mathbf{X}$ & $\mathbf{X}$ & $\mathbf{X}$ & $\mathbf{X}$ & $\mathbf{X}$ & $\mathbf{X}$ \\
\hline Ressignificar as atividades cotidianas (avds e aivds) & & $\mathbf{X}$ & & $\mathbf{X}$ & $\mathbf{X}$ & $\mathbf{X}$ & $\mathbf{X}$ & $\mathbf{X}$ & $\mathbf{X}$ & & $\mathbf{X}$ \\
\hline
\end{tabular}

o tratamento, e sim em estratégias para defesa e manutenção da sua vida.

Embora os profissionais participantes desse estudo não tenham utilizado o termo reabilitação psicossocial, pode-se considerar que os dois objetivos mais citados pelos profissionais, que apresentaram percentual de 90,9 e 72,7, respectivamente, estão em consonância com os princípios desse processo. Para alcançar tais objetivos são adotadas açóes que podem ser caracterizadas como facilitadoras, para que o indivíduo possa assumir sua condiçáa enquanto sujeito social, capaz de exercer sua cidadania.

Barros e Rodrigues (2011, p. 190) refletem que as açóes do terapeuta ocupacional na atenção à pessoa com problemas relacionados ao uso de álcool e outras drogas devem possibilitar

[...] o resgate de capacidades e habilidades perdidas ou 'adormecidas' ao longo do uso crônico de substâncias psicoativas, [...] tendo como foco principal a organização de atividades cotidianas saudáveis.

Refletindo sobre a diversidade encontrada nos processos de intervenção dos profissionais de saúde no atendimento a essas pessoas, Schneider (2010, p. 688) aponta que os serviços são organizados e oferecidos a partir de um referencial teóricometodológico desenvolvido a partir das concepçóes sócio-históricas de todos os envolvidos nesse processo, e que tais concepçóes direcionam "[...] o entendimento sobre o sofrimento, bem como sobre o campo de possibilidades de intervenção na situação de saúde/ doença”.

Essa autora nos apresenta que, segundo os resultados de seu estudo, os serviços de atenção a essa população podem ser categorizados por modelos filosóficos a partir dos quais se concebe o fenômeno da dependência química: Modelo jurídico-moral; Modelo médico ou de saúde pública; Modelo psicossocial; e Modelo sociocultural.

Uma reflexão sobre como alguns desses modelos, aparentemente excludentes e contraditórios, podem ser encontrados em um mesmo serviço é realizada pela pesquisadora a partir dos pressupostos teológicos, metafísicos, políticos e científicos que os sustentam, podendo considerar-se que não existem "[...] contradiçóes em seus fundamentos últimos, na medida em que constituem suas proposiçóes baseados no sincretismo de racionalidades diversas [...]", sendo que 
[...] a maioria dos serviços se equivale nas concepçóes, tanto aqueles que se 'pretendem científicos', quanto aqueles defensores de uma moralidade humana a priori [...]" (SCHNEIDER, 2010, p. 696).

\section{Considerações finais}

Os dados obtidos revelam que os terapeutas ocupacionais que participaram deste estudo realizam intervençôes individuais e grupais. As intervençóes individuais são realizadas duas ou mais vezes por semana por metade dos profissionais. Os outros realizam atendimentos individuais esporadicamente, conforme a demanda expressa pelo usuário do serviço. $\mathrm{O}$ atendimento grupal ocorre principalmente com frequência de duas vezes por semana ou mais.

As atividades mais utilizadas pelos profissionais nos atendimentos foram, em primeiro lugar, as atividades categorizadas como atividades autoexpressivas; em segundo lugar, as atividades manuais, de artesanato e de reciclagem de materiais; e em terceiro lugar as atividades relacionadas à educação em saúde, autocuidado e reorganização do cotidiano.

Os objetivos das atividades propostas mais relatados por esses profissionais foram, em primeiro lugar, resgatar valores pessoais e sociais; e, em segundo lugar, ressignificar as atividades cotidianas, tanto as atividades básicas da vida diária como as atividades instrumentais da vida diária. Em terceiro lugar encontra-se ampliar a corresponsabilizaçáo da família no tratamento; expressar sentimentos e conflitos internos; e usufruir de momentos de lazer e relaxamento, diminuindo a ansiedade e a irritabilidade.

Os profissionais participantes deste estudo apresentaram comportamento bastante diferenciado entre eles, tanto com relação às atividades desenvolvidas com os usuários quanto com relação aos objetivos a serem alcançados. Por exemplo, enquanto os profissionais $\mathrm{P} 3$ e $\mathrm{P} 9$ desenvolvem até seis categorias de atividades, o profissional P8 desenvolve uma. Os objetivos a serem alcançados, listados pelos profissionais, foram agrupados em 15 categorias. Destaca-se que o profissional P9 relatou que espera que o usuário do seu serviço alcance os objetivos que constam em nove categorias, enquanto P2, P5 e P8 relataram objetivos que constam em três categorias, refletindo esse comportamento profissional diferenciado nas intervençôes realizadas.

Reconhece-se os limites deste estudo, visto que os resultados descritos são específicos da amostra apresentada, não sendo passível de generalizações ao contingente de profissionais terapeutas ocupacionais que atuam nessa área. A temática deste estudo apresentou-se de forma desafiadora para as pesquisadoras, que verificam escassa produção de referencial teórico e grande dificuldade na constituição da amostra. Com esta pesquisa não se pretendeu esgotar a temática abordada, sendo assim, cabe ressaltar a necessidade de pesquisas com amostras maiores, tendo este trabalho o papel de iniciar uma longa busca pelo conhecimento sobre a atuação de terapeutas ocupacionais na atenção à pessoa com problemas relacionados ao uso de álcool e outras drogas.

No decorrer do processo de divulgação deste trabalho, vários profissionais contataram as pesquisadoras dispondo-se a contribuir com o estudo, porém não retornaram contato após o envio dos termos do projeto. Houve também alguns que responderam ao instrumento de coleta de dados, mas não devolveram o TCLE assinado e, sendo assim, não puderam ser incluídos. Destaca-se a disponibilidade de três profissionais de Portugal para participar deste estudo, demonstrando a possibilidade de trabalhos conjuntos sobre o tema com pesquisadores daquele país.

Espera-se que o conhecimento obtido por este trabalho sirva como uma fonte de estímulo para o aprimoramento da atuação dos terapeutas ocupacionais inseridos nos espaços destinados à recuperação e reabilitaçáo da pessoa com problemas relacionados ao uso de álcool e outras drogas.

Aponta-se a necessidade de aprofundar a investigação das ações dos terapeutas ocupacionais com essas pessoas, visto que se percebeu neste estudo que ocorre uma falta de sistematização das intervençóes nessa área. Novos estudos podem contribuir para uma maior reflexão e análise $\mathrm{da}$ atuação, por parte dos terapeutas ocupacionais, e a consequente promoçáo da qualidade de vida da população atendida.

\section{Referências}

BRASIL. Ministério da Saúde. Secretaria Executiva. Coordenação Nacional de DST e Aids. A política do Ministério da Saúde para atençâo integral a usuários de álcool e outras drogas. Brasília, 2003.

BALLARIN, M. L. G. S. Abordagens grupais. In: CAVALCANTI, A.; GALVÃO, C. Terapia ocupacional: fundamentação e prática. Rio de Janeiro: Guanabara Koogan, 2007. p. 38-43.

BARDIN, L. Análise de conteúdo. Lisboa: Ediçōes 70, 2010.

BARROS, D. D.; LOPES, R. E.; GALHEIGO, S. M. Projeto Metuia: terapia ocupacional no campo social. $O$ Mundo da Saúde, São Paulo, v. 26, n. 3, p. 365-369, 2002. 
BARROS, M. R. M.; RODRIGUES, M. R. Terapia ocupacional aplicada à dependência química. In: DIEHL, A.; CORDEIRO, D. C.; LARANJEIRA, R. Dependência química: prevenção, tratamento e políticas públicas. Porto Alegre: Artmed, 2011. p. 185-192.

CARVALHO, F. R. M. et al. Causas de recaída e de busca por tratamento referidas por dependentes químicos em uma unidade de reabilitação. Colombia Médica, Cali, v. 42, n. 2, p. 57-62, 2011. Suplemento 1.

CASTRO, E. D.; LIMA, E. M. F. A; BRUNELLO, M. I. B. Atividades humanas e terapia ocupacional. In: CARLO, M. M. R. P.; BARTALOTTI, C. C. Terapia ocupacional no Brasil: fundamentos e perspectivas. São Paulo: Plexus, 2001. p. 41-62.

FLICK, U. Uma introdução à pesquisa qualitativa. Tradução de Sandra Netz. Porto Alegre: Bookman, 2004.

OLIVEIRA, Y. C. A clínica terapêutica ocupacional com usuários de substâncias psicoativas: o desafio da práxis. Revista Brasileira em Promoção da Saúde, Fortaleza, v. 19, n. 4, p. 229-233, 2006. http://dx.doi. org/10.5020/18061230.2006.p229

ORGANIZAÇÃO MUNDIAL DA SAÚDE - OMS; ORGANIZAÇÃO PAN-AMERICANA DA SAÚDE - OPAS. Relatório sobre a saúde no mundo. Saúde mental: nova concepção, nova esperança. Genebra, 2001. ORGANIZAÇÃO MUNDIAL DA SAÚDE - OMS. Neurociências de consumo e dependência de substâncias psicoativas: resumo. Genebra, 2004. 40 p.

SARACENO, B. Libertando identidades: da reabilitação psicossocial à cidadania possível. Rio de Janeiro: Te Corá; Instituto Franco Basaglia, 2001.

SCHNEIDER, D. R. Horizonte de racionalidade acerca da dependência de drogas nos serviços de saúde: implicaçóes para o tratamento. Ciência \& Saúde Coletiva, Rio de Janeiro, v. 15, n. 3, p. 687-698, 2010.

\section{Contribuição dos Autores}

Adriana Maria Nogueira: Participou da concepção do projeto de pesquisa e do instrumento de coleta de dados, coletou e analisou os dados e redigiu o manuscrito. Andrea Ruzzi Pereira: Participou da concepção do projeto de pesquisa e do instrumento de coleta de dados, orientou o desenvolvimento do projeto de pesquisa, da coleta e da análise de dados, realizou a revisão do manuscrito.

\section{Notas}

${ }^{1}$ Este artigo resulta da elaboração do Trabalho de Conclusão de Curso (TCC) do Curso de Terapia Ocupacional da Universidade Federal do Triângulo Mineiro - UFTM; foi apresentado na VI Mostra Científica de Terapia Ocupacional da UFTM e foi aprovado pelo Comitê de Ética em Pesquisa em Seres Humanos da UFTM de acordo com o parecer n⿳亠丷厂 $1920 / 2011$. 